\title{
DIRETRIZES PROJETUAIS DE ILUMINACC̃O PARA ATENDER PESSOAS COM BAIXA VISÃO
}

\section{LIGHTING PROJECT GUIDELINES TO ASSIST PEOPLE WITH LOW VISION}

Giovana Mara Zugliani Bortolan ${ }^{1}$

Marcelo Gitirana Gomes Ferreira ${ }^{2}$

Elton Moura Nickel ${ }^{3}$ 
A ciência e a tecnologia estão inseridas cada vez mais na vida das pessoas. Como meio de incluir pessoas com algum tipo de deficiência, a tecnologia assistiva fornece dispositivos, serviços e estratégias para proporcionar maior autonomia e qualidade de vida. Tendo em vista a falta de estudos que abordem recomendações de iluminação para deficientes visuais, em específico a baixa visão (ou visão subnormal), este artigo tem como objetivo a elaboração de diretrizes de projeto de iluminação para incluir pessoas com baixa visão para que estas possam frequentar ambientes internos com segurança e conforto. Para isso, recorreu-se a revisão sistemática bibliográfica, com o intuito de pesquisar e utilizar as normas, modelos e recomendações de diversos autores e sintetizá-las em um único trabalho, para que seja possível a aplicação destas diretrizes. Permite assim que profissionais possam se basear nestas diretrizes e projetar ambientes para pessoas portadoras de visão subnormal, e que estas possam exercer suas atividades e frequentar ambientes internos e sociais percebendo o espaço de maneira mais plena possível.

Palavras-Chave: diretrizes projetuais, iluminação, ambientes internos, baixa visão.
Science and technology are becoming more embedded in people's lives each year. As a means of including people with some kind of disability, assistive technology provides devices, services and strategies to provide greater autonomy and quality of life. Given the lack of studies that address lighting recommendations for the visually impaired, specifically low vision, this article aims to develop lighting design guidelines to include people with low vision so that they can attend indoors with safety and comfort. For this, a systematic bibliographic review was used to search and use the norms, models and recommendations of several authors and synthesize them in a single work, so that it is possible to apply these guidelines. It allows professionals to be able to base themselves on these guidelines and to design environments for people with low vision, and that these can exercise their activities and attend internal and social environments perceiving the space as fully as possible.

Keywords: project guidelines, lighting, interior, low vision.

\footnotetext{
1 Graduada em Licenciatura em Educação Artística com Habilitação em Artes Plásticas pela Universidade Estadual Paulista Júlio de Mesquita Filho, Faculdade de Arquitetura, Artes e Comunicação, Campus Bauru. Possui técnico de nível médio em Design de Interiores, na instituição de ensino Senac, campus Bauru. Atualmente é aluna regular do programa de pós graduação de Design da Universidade do Estado de Santa Catarina, campus Florianópolis, com área de concentração em Métodos para fatores humanos na linha de pesquisa Interfaces e interações físicas.

gmzugliani@gmail.com

\begin{abstract}
2 Professor Associado do Departamento de Design da UDESC, atuando na graduação do curso de Design, nas pós-graduações em Design (atual coordenador do PPGDesign) e em Administração desta universidade, assim como no curso de pós-graduação em Engenharia de Produção da UFSC. Tem experiência na área de Engenharia Mecânica e de Produção, com ênfase em Desenvolvimento de Produtos, atuando principalmente nos seguintes temas: ergonomia e usabilidade, e desenvolvimento de TAs, metodologia de projeto, desenvolvimento colaborativo de produtos, sustentabilidade no projeto. Possui graduação em Engenharia Mecânica pela UFPE (1992). Realizou mestrado e doutorado em Engenharia Mecânica na UFSC (1997 e 2006), na área de Projeto de Sistemas Mecânicos. Fez estágio de pós-doutorado em Design Cerâmico na A2D (Agência para o Design Cerâmico) da UFSC em 2008. Trabalhou como engenheiro de projeto mecânico na Rhodia Nordeste (1992 a 1994) e como engenheiro de produto da Volkswagen-Audi em Curitiba (1998 a 2002). Foi engenheiro residente na sede da Volkswagen, em Wolfsburg na Alemanha (1999 a 2000).

Universidade do Estado de Santa Catarina
\end{abstract} marcelo.gitirana@gmail.com
}

3 É Doutor (2012) e Mestre (2009) em Engenharia de Produção pela Universidade Federal de Santa Catarina, na área de Engenharia do Produto e Processo e possui graduação em Design Industrial pela Universidade do Estado de Santa Catarina (2006). Atualmente é Chefe do Departamento de Design e Professor Permanente do Programa de Pós-Graduação em Design da Universidade do Estado de Santa Catarina.

eltonnickel@gmail.com 


\section{Introdução}

O Censo (2012) define a deficiência como um tema de direitos humanos que obedece ao princípio de que todo ser humano tem o direito de desfrutar de todas as condições necessárias para o desenvolvimento de suas habilidades e capacidades, sem ser submetido a qualquer tipo de discriminação. Para Bonatti et al. (2007), a deficiência é o desvio significativo ou perda de função nas estruturas do corpo, enquanto que funcionalidade abrange todas as funções do corpo como atividade e participação.

Com o aumento da expectativa de vida, cresce o número de indivíduos que podem apresentar problemas de baixa visão, incluindo os casos de origem congênita. Estes representam uma parcela significativa da população que a cada dia vem se inserindo de maneira mais ativa na sociedade, requisitando que os serviços e produtos oferecidos também privilegiem suas características perceptivas, uma vez que são usuários potenciais dos espaços sociais e culturais (ESCOBAR, 2011).

Ambientes internos sociais devem além da acessibilidade para pessoas com deficiência de mobilidade, proporcionar conforto e segurança a pessoas com baixa visão, que em certos ambientes sofrem com brilho excessivo, iluminação escassa, baixo contraste, entre outros problemas. Fresteiro $(2005$, p. 191) pontua que a iluminação é um elemento que define os espaços, sempre guiada pelas normas, porém estas quase sempre são elaboradas "para as necessidades de pessoas com visão normal ou sem problemas de mobilidade, e poucas vezes é utilizada para sinalizar corretamente os obstáculos que apresentam os espaços".

Segundo Faia (2013) a maioria dos estatutos, regulamentos e orientações consideram pessoas com deficiência visual, porém não abordam a baixa visão. É preciso buscar e desenvolver orientações para o design de ambientes, garantir a segurança da população crescente de pessoas com visão subnormal e a capacidade dessas orientações funcionarem em todos os tipos de construção. Fresteiro (2005) também alega a falta de materiais e estudos referentes a parâmetros de iluminação para pessoas com baixa visão. Encontram-se em manuais e recomendações de acessibilidade formas de construir e facilitar a mobilidade de pessoas com visão subnormal, "mas a iluminação não é levada em consideração" (FRESTEIRO, 2005, p.1012).

Riazi et al. (2012) apontam que existem muitas verificações e diretrizes para pessoas com deficiência visual, porém tendem a ser excessivamente generalizadas e pouca pesquisa empírica citada. "A maioria se não todas as orientações não fazem distinção entre as diferentes causas e características da deficiência visual e, como consequência, as diferentes intervenções necessárias" (RIAZI et al., 2012. p. 271).

Tural e Tural (2014) afirmam que a literatura sobre sistema visual de envelhecimento e doenças oculares relacionadas à idade são detalhadas e bem definidas. Além disso, as pesquisas sobre as relações entre os níveis de iluminação e funções visuais em oftalmologia são vastos, mas a pesquisa voltada para arquitetura e design de ambientes referentes a diretrizes de iluminação e ferramentas de avaliação visual à base de percepção são limitadas. Portanto, torna-se necessária a exploração para a melhoria de ambientes luminosos para neutralizar problemas referentes à deficiência visual.

Desse modo, o artigo apresenta um estudo sobre a deficiência visual, em específico a baixa visão em relação à iluminação artificial de ambientes internos, e busca 
apresentar um conjunto de diretrizes para projetos de iluminação para incluir pessoas com baixa visão. Seguindo o objetivo de Fresteiro (2005, p. 191), não cabe aqui desenvolver uma teoria específica para um grupo especial, mas utilizar os diferentes modelos e recomendações para a elaboração de um projeto no qual "o indivíduo se encontre suficientemente cômodo para exercer suas atividades, percebendo o espaço de maneira mais plena possível".

Projetar um sistema único de iluminação que responda aos múltiplos requerimentos pode ser utópico. Porém como afirma Fresteiro (2010, p. 272) "é importante levar em conta que observando algumas regras básicas pode-se conseguir um bom projeto que favoreça a maioria dos possíveis usuários", para que as capacidades de pessoas com baixa visão possam ser ampliadas.

Nos próximos itens serão abordados os temas: baixa visão (individualizando os tipos mais comuns) e projetos de iluminação para estes usuários. Em seguida, será apresentado o resultado do estado da arte das recomendações de acordo com as diretrizes projetuais citadas e pesquisadas para atender pessoas com baixa visão.

O método utilizado para atingir o objetivo proposto foi a revisão sistemática bibliográfica (RBS) que se trata de uma técnica bastante difundida principalmente nas áreas de Medicina, Psicologia e Ciências Sociais e, se realizada com rigor e de forma estratégica, permite atingir elevados níveis de confiabilidade nos resultados (CONFORTO et al., 2011).

Através das palavras chaves: diretrizes projetuais, iluminação, ambientes internos e baixa visão, foram encontrados artigos, teses, dissertações e capítulos de livros, obtendo assim uma base sólida para o desenvolvimento das diretrizes projetuais.

\section{Baixa Visão}

O Art. 2 da Lei $n \cong$ 38/2004 considera a pessoa com deficiência aquela que por motivo de perda ou anomalia, congênita ou adquirida apresenta dificuldades específicas susceptíveis de limitar ou dificultar sua atividade e participação em condições de igualdade com as demais pessoas (BRASIL, 2004). Os dados coletados pelo Instituto Brasileiro de Geografia e Estatística - IBGE, no censo demográfico de 2010, apontaram que $23,9 \%$ da população total têm algum tipo de deficiência - visual, auditiva, motora e mental ou intelectual. A deficiência visual apresentou a maior ocorrência, afetando $18,6 \%$ da população brasileira, e teve maior prevalência em todos os grupos de idade, sendo bastante acentuada no grupo acima de 65 anos, ocorrendo em quase metade da população desse segmento $(49,8 \%)$.

A informação visual é responsável por uma parte considerável da apreensão da realidade. Esta constitui, portanto, um sentido especial uma vez que é responsável por transmitir informação detalhada de objeto a grandes distâncias (COSTA, 2012). "A visão é responsável por $85 \%$ do contato do indivíduo com o mundo" (BONATTI et al., 2007, p. 196), ou seja, é o canal mais importante de relacionamento deste indivíduo com o mundo exterior. Assim como a audição, ela capta registros próximos ou distantes e permite organizar, no nível cerebral, as informações trazidas pelos outros órgãos dos sentidos (GIL, 2000).

A deficiência visual impede os mecanismos que favorecem a informação, comunicação e mobilidade que são condições necessárias para a independência e autono- 
mia de todas as pessoas (FRESTEIRO, 2005). O vidente percebe sem esforço todo o espaço. A pessoa com deficiência deve fazer a síntese de sua exploração, que têm um caráter analítico e sequencial, portanto significa muito mais para um deficiente visual que os obstáculos sejam eliminados à realizar alterações nos espaços já construídos (ESCOBAR, 2011).

A Organização Mundial de Saúde (OMS) considera deficiente visual a pessoa que é privada, em parte (segundo critérios pré-estabelecidos) ou totalmente, da capacidade de ver. Segundo o Art. 5어 do Decreto n 5.296/04 de 2 de dezembro de 2004 da Presidência da República, Deficiência Visual é:

Cegueira, na qual a acuidade visual é igual ou menor que 0,05 no melhor olho, com a melhor correção óptica; a baixa visão, que significa acuidade visual entre 0,3 e 0,05 no melhor olho, com a melhor correção óptica; os casos nos quais a somatória da medida do campo visual em ambos os olhos for igual ou menor que $60^{\circ}$; ou a ocorrência simultânea de quaisquer das condições anteriores (BRASIL, 2004, p.1).

Gil (2000) define baixa visão como a alteração da capacidade funcional decorrente de fatores como rebaixamento significativo da acuidade visual, redução importante do campo visual e da sensibilidade aos contrastes e limitação de outras capacidades. O MEC (2006) em seu manual de saberes e práticas da inclusão, complementa a informação com: alterações corticais que interferem ou que limitam o desempenho visual do indivíduo. A acuidade visual é a clareza ou a nitidez da visão, já o campo de visão é a área que uma pessoa pode ver quando seus olhos estão fixos em uma posição (ALLAN, KIRKPATRICK e HENRY, 2016).

A perda da função visual pode se dar em nível severo, moderado ou leve, podendo ser influenciada também por fatores ambientais inadequados. Dentre os grupos de pessoas com visão subnormal também há variações. Alguns podem ter dificuldade de realizar as tarefas diárias como: ler frascos de medicamentos, reconhecer rostos familiares, além do aumento do risco de queda, ameaçando a autonomia. Outros, porém, conseguem ler se o impresso for grande ou se estiver próximo a seus olhos (ou mesmo através de lentes de aumento), ou detectar grandes formas, cores e contrastes (SONZA, 2013).

A baixa visão não pode ser corrigida com óculos, cirurgia ou outro tratamento médico. Isto não significa que as pessoas com baixa visão têm dificuldade na realização de tarefas, mas que sua qualidade de vida e bem-estar podem ser afetados (BRUNNSTRÖM et al., 2004). A principal causa da visão subnormal é de origem genética, como retinite pigmentosa e gestação precoce nos casos de: uso de drogas, álcool, infecções durante a gravidez como rubéola, sífilis, AIDS, toxoplasmose, doenças virais e bacterianas como sarampo, meningites e encefalites (MEC, 2006). As doenças oculares e condições de saúde como catarata, glaucoma e diabetes que são mais prevalentes em pessoas idosas (ALLAN, KIRKPATRICK e HENRY, 2016; BAKKER, 2005). 


\subsection{Tipos de baixa visão e suas características}

São várias as doenças oculares que podem provocar alterações significativas na visão. Segundo o centro especializado em baixa visão da Europa (BAJA VISIÓN ÁNGEL BARAÑANO, 2016) existem sete tipos de baixa visão, e estão dispostos na figura 1 a seguir:

Figura1 - Doenças comuns da baixa visão
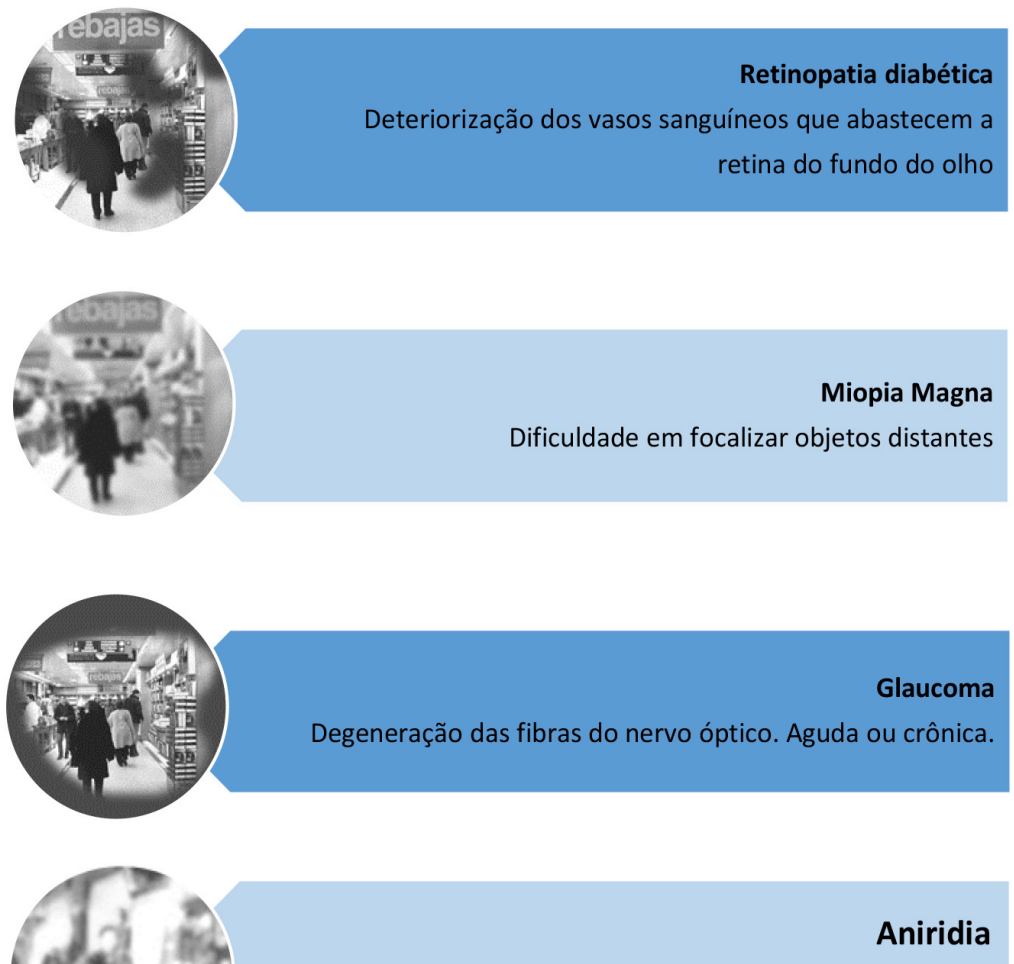

Falta total ou parcial da íris
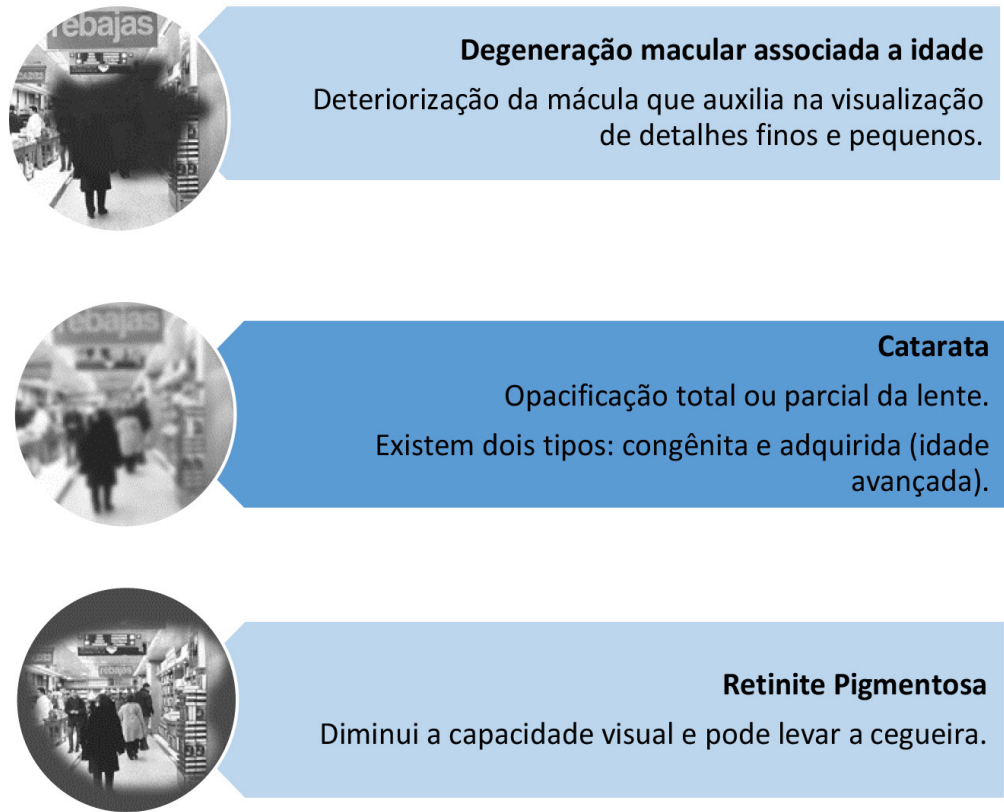

Fonte: Adaptado do centro especializado em baixa visão da Europa (2016) 
Os sintomas da degeneração macular envolvem perda da nitidez, distorção de linhas, problemas na visualização de detalhes. Já a catarata causa perda do foco de luz e produção de imagens claras, pouco nítidas e névoa (película) sobre os olhos. A miopia além de dores de cabeça, irritação nos olhos e desconforto visual provoca perda da visão de objetos extremamente próximos ou perda de visão à distância, além de visão distorcida. Manchas na frente da visão, defeitos no campo visual, falta de visão periférica são sintomas mais comuns da retinite pigmentosa, glaucoma e da retinopatia diabética. Já a sensibilidade anormal à luz ou claridade excessiva são sintomas comuns da aniridia (MEC, 2006).

\subsection{Projeto de iluminação de ambientes para pessoas com baixa visão}

Segundo a European Standarts (EN 12464-2, 2007) o objetivo da iluminação é a obtenção de boas condições de visão associadas à visibilidade, segurança e orientação dentro de um determinado ambiente. Além de atender aos aspectos qualitativos, que garantem o bem-estar psicológico e bom funcionamento fisiológico dos usuários, a iluminação está relacionada à produção hormonal que afeta diretamente o estado de ânimo e a produtividade das pessoas.

A boa iluminação é muito mais que apenas fornecer uma boa visualização de uma atividade, é essencial que as atividades sejam realizadas facilmente e com conforto. Em geral a iluminação deve assegurar: conforto visual, dando as pessoas uma sensação de bem-estar; desempenho visual, de modo que estejam capacitados de realizar suas atividades de forma precisa e com segurança (ABNT, 2013). Além disso, deve ser encarada como "um dos fatores primordiais para a inclusão de pessoas que têm percepção e apreensão do ambiente construído fora do convencional" (FRESTEIRO, 2005, p. 191).

O controle da iluminação artificial pode diminuir o desconforto visual, com o aumento do contraste e da resolução visual. Pessoas com baixa visão necessitam de níveis diferentes de iluminação. Alguns podem apresentar maior sensibilidade em ambientes com maior intensidade luminosa enquanto outros, menor sensibilidade ao contraste, necessitando de períodos prolongados para adaptação fotópica ou escotópica ${ }^{1}$.

No caso de idosos, estes necessitam de três vezes mais iluminação que um indivíduo de vinte anos para a realização das mesmas atividades (MIZIARA, 2015). A visão depende da presença de luz, mas pode depender do estímulo visual e das características do sistema visual individual. Bakker (2013) faz uma observação quanto às pessoas que possuem tipos de baixa visão semelhantes e que podem responder de formas diferentes aos diversos níveis de luz.

Para que a iluminação seja confortável, deve-se experimentar diversos tipos de lâmpadas e intensidades. "A qualidade da luz é tão importante quanto a quantidade (...) é por isso que não deve apenas substituir as lâmpadas de baixa potência com lâmpadas de maior potência pois só aumentará o brilho" (BAKKER, 2013, p. 11). A direção da luz deve ser adequada, em conjunto com as condições ideais de luminosidade, $1 \quad$ O olho tem dois tipos de células fotossensíveis ou fotorreceptores sensíveis a luz que são os cones e os bastonetes. A maior quantidade de cones encontra-se na fóvea, que é uma zona localizada na parte central do fundo da retina, e na parte periférica da retina só existem bastonetes. Os cones são mais sensíveis à iluminação mais intensa e encarregados da visão das cores (visão fotópica). Os bastonetes são acromáticos, ou seja, não distinguem cores (visão escotópica), só vem imagens em branco e preto, mas apenas formas, são mais sensíveis a baixos níveis de energia da luz e estão mais dispersos na retina (LAUAR, 2012). 
controle de brilho e a escolha certa dos acessórios para a iluminação (BRUNNSTRÖM et al., 2004).

Andrade (2013) informa que a iluminação deve ser uniforme evitando a desorientação das pessoas que precisam de mais tempo para se adaptar às mudanças na luz. O autor também alerta sobre a importância de haver contrastes de cor entre o chão e as paredes. Faia (2013, p.2) propõe que em construções universalmente acessíveis, deve haver uma iluminação controlada para evitar ou minimizar o brilho de objetos que são visualizados e devem ser distinguíveis através de sombras, cores, texturas e contraste.

Fresteiro (2005) descreve que um dos grandes problemas que as pessoas com baixa visão encontram ao entrar em ambiente interno é o tempo de adaptação a um nível diferente de iluminação, impedindo-as de detectar um possível obstáculo, bem como a intensidade da luz que estas requerem, pois mudanças de luminosidade podem levar à perda total de qualquer referência. Para explorar e fazer uma síntese do espaço, a pessoa com deficiencia visual necessita de estratégias sequenciais para obter uma representação do local.

Andrade (2003) explica que os níveis de iluminação adequados às pessoas com baixa visão devem considerar parâmetros muito mais amplos do que para as pessoas com visão normal, e adaptações individuais são necessárias, considerando impacto que as patologias individuais causam. O autor também alerta para a importancia na concepção de espaços em que todos os aspectos estão envolvidos em conjunto com a fonte de luz utilizada como: contraste, tamanho e localização de luminárias. Wright, Hill e Cook (1999) complementam que os requisitos de iluminação para qualquer tarefa necessitam de informações sobre o indivíduo, a patologia ocular, a tarefa a ser realizada e o ambiente em geral.

\section{Medotologia}

Para o desenvolvimento do presente artigo, foi utilizada como metodologia a revisão sistemática bibliográfica (RSB) que segundo Ferenhof e Fernandes $(2016$, p. 2) é um método de investigação científica "com um processo rigoroso e explícito para identificar, selecionar, coletar dados, analisar e descrever as contribuições relevantes a pesquisa".

Para nortear a revisão sistemática, foi definido o seguinte problema: Como iluminar ambientes de forma adequada para incluir pessoas com baixa visão? Assim, foram definidas as palavras chaves: diretrizes projetuais, iluminação, ambientes internos e baixa visão, que foram utilizadas para a realização do levantamento bibliográfico para atender o objetivo deste artigo, sendo este: organizar e construir um pensamento consolidado para guiar projetos de iluminação para atender pessoas com baixa visão.

Antes de iniciar a RBS, foram realizadas buscas exploratórias (pesquisa isolada, sem os critérios específicos utilizados na revisão sistemática) e foram encontrados para o desenvolvimento do presente artigo seis documentos expressivos e uma homepage. Estas referências (demonstradas na tabela 1) foram julgadas como relevantes, pois condizem com o tema abordado e contém dados imprescindíveis para o desenvolvimento das diretrizes sendo complementares à revisão sistemática que será mostrada na tabela 4 . 


\begin{tabular}{|c|c|c|c|}
\hline Tema & Título & Autores & Tipo/Ano \\
\hline Baixa visão/diretrizes & Architecture for low vision & FAIA, S. L. K. & Artigo/2013 \\
\hline \multirow{2}{*}{$\begin{array}{l}\text { Baixa visão/ } \\
\text { Iluminação }\end{array}$} & $\begin{array}{l}\text { O conservador em conflito: a } \\
\text { iluminação em museus com } \\
\text { proposta para inclusão de pessoas } \\
\text { com deficiência visual. }\end{array}$ & ESCOBAR, G. & $\begin{array}{l}\text { Trabalho acadêmico } \\
\text { Mestrado/ } 2011\end{array}$ \\
\hline & $\begin{array}{c}\text { Household Tips for People with } \\
\text { Low Vision }\end{array}$ & BAKKER, R. & Artigo/2005 \\
\hline \multirow[t]{2}{*}{$\begin{array}{l}\text { Acessibilidade/ } \\
\text { Baixa visão }\end{array}$} & $\begin{array}{l}\text { A influência da iluminação nos } \\
\text { ambientes acessíveis: } \\
\text { identificando barreiras }\end{array}$ & FRESTEIRO, R.H & $\begin{array}{l}\text { Capítulo de } \\
\text { livro/2010 }\end{array}$ \\
\hline & $\begin{array}{l}\text { Iluminação inclusiva, ou } \\
\text { eliminando barreiras lumínicas }\end{array}$ & FRESTEIRO, R.H & $\begin{array}{l}\text { Capítulo de } \\
\text { livro/2005 }\end{array}$ \\
\hline $\begin{array}{l}\text { Acessibilidade/ } \\
\text { Iluminação }\end{array}$ & $\begin{array}{l}\text { La iluminación de los espacios } \\
\text { como parámetro de acessibilidade } \\
\text { para personas con baja visión }\end{array}$ & FRESTEIRO, R.H & $\begin{array}{c}\text { Tese de } \\
\text { doutorado/2002 }\end{array}$ \\
\hline
\end{tabular}

Para a revisão sistemática bibliográfica, a base de dados utilizada foi a Scopus, uma plataforma que oferece um conjunto de bases de dados para estudo e análise de citações de artigos, publicações, autores e agências de fomento. Para iniciar a pesquisa na base de dados, foram definidos os seguintes critérios para seleção dos artigos: determinação das palavras-chaves, seleção de artigos recentes (últimos vinte anos); leitura de títulos, abstracts, leitura diagonal (introdução, conclusão e métodos) e leitura completa do artigo. Os critérios de seleção foram organizados na tabela a seguir (Tabela 2).

Tabela 2- Síntese de Métodos e Técnicas utilizadas

\begin{tabular}{ll}
\multicolumn{1}{c}{ Métodos e técnicas de pesquisa } & \multicolumn{1}{c}{ Resultados } \\
\hline $\begin{array}{l}\text { Determinação das palavras chaves no idioma inglês: } \\
\text { methods, visual discomfort, workplaces, artificial } \\
\text { lighting }\end{array}$ & $\begin{array}{l}\text { Organização e combinação das palavras chaves, } \\
\text { a fim de reduzir o número de artigos. }\end{array}$ \\
\hline $\begin{array}{l}\text { Organização dos artigos } \\
\text { Selecionados os artigos dos últimos vinte anos } \\
\text { (dentro do tema proposto) }\end{array}$
\end{tabular}




\begin{tabular}{|l|l} 
Seleção dos artigos & Por título e palavras chaves \\
\hline Critérios de exclusão & Foram retirados os artigos fora do contexto da \\
& $\begin{array}{l}\text { pesquisa, sem cunho científico, com mais de } \\
\text { vinte anos de publicação e os que não possuíam } \\
\text { acesso gratuito. }\end{array}$
\end{tabular}

Fonte: Os autores (2016)

A pesquisa foi realizada entre os dias 19 de outubro a 26 de outubro de 2016. A busca gerou na primeira pesquisa, 30 documentos, e nesta etapa não foram aplicados filtros. Após a segunda triagem, considerando os critérios de seleção (tabela 2), os resultados foram de 16 documentos.

Posteriormente, foram analisados os resumos, sendo retirados da pesquisa aqueles que não se adequavam em função de seu tema e de sua abordagem. Resultaram apenas quatro documentos, e os mesmos foram utilizados para a revisão sistemática do assunto a ser explorado. Os documentos utilizados na revisão metodológica foram eleitos conforme sua relevância para a pesquisa, considerando principalmente a qualidade dos textos e o seu tema, e estão descritos na tabela 3.

Tabela 3 - Resultados da seleção de pesquisa na base de dados Scopus

\begin{tabular}{l|c|c|}
\hline \multicolumn{1}{|c}{ Título do documento } & Autores & $\begin{array}{c}\text { Ano de } \\
\text { publicação }\end{array}$ \\
\hline $\begin{array}{l}\text { Quality of light and quality of life - the effect of lighting } \\
\text { adaptation among people with low vision }\end{array}$ & Brunnström et al. & 2004 \\
\hline $\begin{array}{l}\text { Home modification guidelines as recommended by } \\
\text { visually impaired people }\end{array}$ & Riazi et al. & 2012 \\
\hline $\begin{array}{l}\text { Office task lighting: A user study of six tasks lights by five } \\
\text { workers with low vision }\end{array}$ & Wright, Hill e Cook & 1999 \\
\hline $\begin{array}{l}\text { Luminance contrast analyses for low vision in a senior } \\
\text { living facility: A proposal for na HDR image-based } \\
\text { analysis tool }\end{array}$ & Tural e Tural & 2014 \\
\hline
\end{tabular}

Fonte: Os autores (2016)

\section{Resultados e Discussão}

Os espaços internos devem ser visíveis para pessoas com baixa visão, por ter contrastes adequados e brilho mínimo de luz natural ou artificial (FAIA, 2013; BRUNNSTRÖN et al., 2004). Deve-se evitar iluminação direta, pois apesar de ser um sistema eficaz, que proporciona luz pontual, tem o inconveniente de produzir sombras e ofuscamentos diretos e reflexivos (FRESTEIRO, 2005).

Em espaços de circulação (entradas e corredores), devem ser evitados obstáculos perigosos, como móveis, colunas e objetos a menos que haja contraste de luz para distingui-los dos arredores (FAIA, 2013); para que os olhos se adaptem de um espaço para o outro: caminhos e passarelas devem ser bem iluminados a noite (BAKKER, 2013); prever linhas de luz no centro ou na lateral de corredores como um guia. Principalmente em entradas deve-se evitar obstáculos no centro, caso seja impossível, deverão ser sinalizados com iluminação de destaque e um contraste diferente (FRESTEIRO, 2005). 
Iluminação ao longo de passagens deve ser uniforme, e a utilização de sensores de movimento e temporizadores para que haja menos necessidade de a pessoa localizar um interruptor de luz para ligar as luzes e desligá-las (RIAZI, et al., 2012). Escadas, rampas e degraus devem ter uma iluminação uniforme que cubra toda a área (FRESTEIRO, 2005); nível ideal de escadas para pessoas com deficiência visual é de 500 lux, para rampas 300 lux (ESCOBAR, 2011). Inclusão de contrastes de cores entre o corrimão e as paredes e maior distribuição de luminância para a distinção (TURAL e TURAL, 2014).

Para iluminações de parede, deve haver interruptores de três vias para que possa ligar e desligar a luz (BAKKER, 2013). No caso de luminárias e superfícies brancas exceto as que dirigem o fluxo para o teto, não são geralmente indicadas para as pessoas com visão subnormal porque proporcionam uma iluminação desigual e às vezes ofuscamento, a não ser que se utilizem elementos difusores para ajudar na orientação (FRESTEITO, 2005). Quanto as cores, deve haver um contraste se houver superfícies brancas e iluminação branca (FAIA, 2013). Optar por cores claras para evitar adaptação visual e escura para ter contraste, pois, serve de localização e identificação (FRESTEIRO, 2005).

Os contrastes são necessários para visualização de objetos, portas, paredes e móveis (RIAZI, et al., 2012). O brilho é um problema para muitas pessoas, não só causa desconforto no olhos mas também interfere na capacidade de visualização. Utilizar luminárias com lâmpadas que proporcionem ao ambiente luz uniforme e os acabamentos do espaço e móveis devem ser foscos e não polidos. (BAKKER, 2013); em janelas deve ser controlado com sombreamento (FAIA, 2013). Em caso de superfícies brilhantes, não ter foco de luz sobre elas (ESCOBAR, 2011).

Pisos, paredes e mobiliários com brilho em geral causam reflexos e produzem ofuscamento. Deve-se preferir superfícies foscas a muito polidas (FRESTEIRO, 2005). Em grandes espaços, as luminárias devem ser uniformemente colocadas. Se houver iluminação natural deve ser aproveitada desde que não se situe dentro do campo visual da pessoa, pois pode produzir ofuscamento (final de escada ou corredor). Deve-se evitar cortinas (FRESTEIRO, 2005). Nível ideal para pessoas com deficiência visual é de 300 lux (ESCOBAR, 2011).

Quanto a espaços que recebem diversas pessoas deve-se evitar iluminação que cause ofuscamento, afinal pode afeta qualquer pessoa independente da condição visual (FRESTEIRO, 2005). Espaços de trabalho devem ter atenção a iluminação direcionada em monitores de computador que ocasionam ofuscamento, não produzir brilhos e ter uma distribuição uniforme (FRESTEIRO, 2005). Aumento de 300-350 lux em relação ao mínimo de luz, onde a pessoa precisa de mais atenção e 580 lux em espaços de trabalho (Brunnström et al., 2004); usar luz para uma tarefa é melhor que não usar, mesmo estas que fazem sombra.

Para luminárias flexíveis e ajustáveis deve-se instalar interruptores e dimmers para controlar a quantidade de luz (WRIGHT, HILL e COOK, 1999; BAKKER, 2013). Os espaços com pé direito baixo devem evitar reflexos da luz no campo visual (FRESTEIRO, 2005). Para iluminação geral utilizar luminárias de mesa alta, com tons claros e abas largas na parte inferior ou torchieres (Figura 2), utilizar lâmpadas de 150-300 watts, dependendo do tamanho do ambiente (BAKKER, 2013). 
Figura 2: Iluminação com torchieres que pode ser utilizada como iluminação geral.

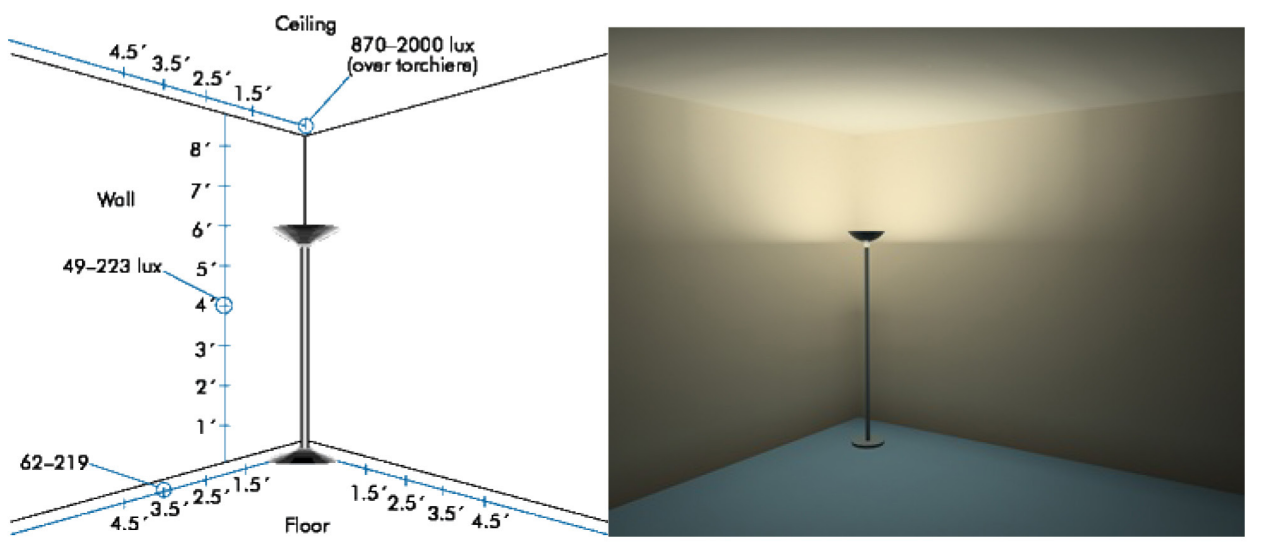

Fonte: Light Research center (2017)

Já a iluminação para uma tarefa detalhada deve-se investir em uma lâmpada de qualidade com um "pescoço" flexível (Figura 3) permitindo que o usuário mova a luz para baixo e perto da sua tarefa. Para reduzir o brilho, certifique-se que a lâmpada esteja abaixo do nível do olho; para iluminação de tarefa/leitura é recomendado uma lâmpada de 60 watts colocado a $30 \mathrm{~cm}$ longe da tarefa (BAKKER, 2013).

Figura 3: Luminária com pescoço flexível que pode ser utilizada como iluminação para tarefa detalhada.

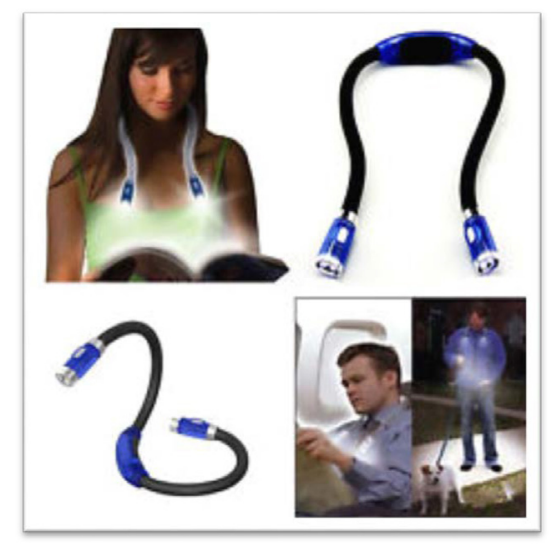

Fonte: Ebay (2017)

Iluminações ajustáveis devem utilizar sistema de regulagem de intensidade ou dimerização que permite um controle flexível e auto ajustável, ou deve-se prever a iluminação complementar com luminárias individuais. Porém atenção ao "efeito zebra" (zona de sombra intercalada com zona de luz) pois, desorienta pessoas produzindo confusão e insegurança nos deslocamentos (FRESTEIRO, 2005).

Quanto à iluminação direta deve-se ressaltar os detalhes que sejam de interesse, tais como escadas, cartazes, números, painéis, indicativos, plantas, utilizando luzes diretas sobre eles, sem produzir reflexos para que possam ser localizados com maior facilidade (FRESTEIRO, 2005). Os níveis devem ser de 500 lux (ESCOBAR, 2011). Já a iluminação semi-direta, que não incide em sua totalidade em uma área determinada e proporciona menos sombras e ofuscamentos é preferível (FRESTEIRO, 2005). 
Iluminação branca como lâmpadas fluorescentes de espectro total e LED oferecem luz mais confortável de tarefas para pessoas com baixa visão. Esta luz branca fria (com um tom azulado) é excelente para tarefas visuais porque realça cores e contraste, principalmente para os indivíduos com degeneração macular (BAKKER, 2013); luz branca oferece maior conforto; fluorescente proporciona iluminação geral difusa para pessoas com deficiência visual e enfatiza as cores que contém azul (FRESTEIRO, 2005). Fontes fluorescentes são as mais indicadas, pois proporcionam uma iluminação uniforme (WRIGHT, HILL e COOK, 1999).

\subsection{Diretrizes projetuais}

\section{Abaixo (tabela 4) seguem as diretrizes projetuais recomendadas pelos autores citados na revisão sistemática bibliográfica, discutidas no item 5 .}

Tabela 4 - Diretrizes proietuais de iluminacão para pessoas com baixa visão.

\begin{tabular}{ll}
\hline Espaços & Espaços internos \\
internos e de & Deve ser visível, com contrastes adequados e brilho mínimo de luz natural ou artificial \\
circulação & (FAIA, 2013; BRUNNSTRÖN et al, 2004) Deve-se evitar iluminação direta \\
& (FRESTEIRO, 2005).
\end{tabular}

Espaços de circulação (entradas e corredores)

Devem ser evitados móveis, colunas e objetos a menos que haja contraste de luz para distingui-los dos arredores (FAIA, 2013). Caminhos e passarelas devem ser bem iluminados a noite e linhas de luz no centro ou na lateral de corredores como um guia (BAKKER, 2013). Utilização de sensores de movimento e temporizadores (RIAZI, et al., 2012). Linhas de luz no centro ou na lateral de corredores, como um guia e evitar obstáculos no centro (FRESTEIRO, 2005).

\section{Escadas, rampas e degraus}

Interruptor de três vias para que possa ligar e desligar a luz. As escadas, rampas e degraus devem ter iluminação uniforme que cubra toda a área (BAKKER, 2013). Nível ideal de escadas para pessoas com deficiência visual é de 500 lux, para rampas 300 lux (ESCOBAR, 2011). Inclusão de contrastes de cores entre o corrimão e as paredes (TURAL e TURAL, 2014).

\section{Bilho e} luminárias

\section{Luminárias de parede}

Devem ser evitadas, por causar desorientação, exceto as que dirigem o fluxo para o teto (BAKKER, 2013)

\section{Luminárias e superfícies brancas}

Deve haver contraste se houver superficies brancas e iluminação branca porque proporcionam uma iluminação desigual e às vezes ofuscamento, a não ser que se utilizem elementos difusores para ajudar na orientação (FRESTEITO, 2005). Deve haver um contraste se houver superficies brancas e iluminação branca (FAIA, 2013).

\section{Brilho}

Utilizar luminárias com lâmpadas que proporcionem ao ambiente luz uniforme e os acabamentos do espaço e móveis devem ser foscos e não polidos (BAKKER, 2013); em janelas deve ser controlado com sombreamento. Em caso de superfícies brilhantes, não ter foco de luz sobre elas (ESCOBAR, 2011). Pisos, paredes e mobiliários brilhantes em geral causam reflexos e produzem ofuscamento. Deve-se preferir superfícies foscas a muito polidas (FRESTEIRO, 2005). 
Cores e contrastes

\section{Cores}

Optar por cores claras para evitar adaptação visual e escuras para ter contraste pois serve de localização e identificação (FRESTEIRO, 2005).

\section{Contrastes}

São necessários para visualização de objetos, portas, paredes e móveis (RIAZI, et al., 2012).

\section{Grandes} espaços e espaços de trabalho

\section{Em grandes espaços}

Luminárias uniformemente colocadas (FRESTEIRO, 2005). Se houver iluminação natural deve ser aproveitada desde que não se situe dentro do campo visual da pessoa pois pode produzir ofuscamento (final de escada ou corredor). Deve-se evitar cortinas (FRESTEIRO, 2005). Nível ideal para pessoas com deficiência visual é de 300 lux, (ESCOBAR, 2011), exceto as escadas que exige maior atenção (ESCOBAR, 2011).

\section{Espaços com pé direito baixo}

Evitar reflexos da luz no campo visual (FRESTEIRO, 2005).

\section{Espaço de trabalho}

Atenção à iluminação direcionada em monitores de computador que ocasionam ofuscamento (FRESTEIRO, 2005). Não produzir brilhos e ter uma distribuição uniforme (FRESTEIRO, 2005). Aumento de 300-350 lux em relação ao mínimo de luz, onde a pessoa precisa de mais atenção e 580 lux em espaços de trabalho (Brunnström et al, 2004). Faz-se necessário uso de luminárias flexíveis e ajustáveis (WRIGHT, HILL e COOK, 1999), instalação de interruptores e dimmers para controlar a quantidade de luz (BAKKER, 2013).

\section{Iluminação: geral, detalhada e direta}

\section{Iluminação geral}

Utilizar luminárias de mesa alta, com tons claros e abas largas na parte inferior ou "torchieres", utilizar lâmpadas de 150-300 watts, dependendo do tamanho do ambiente (BAKKER, 2013).

\section{Iluminação de tarefa detalhada}

Investir em lâmpada de qualidade com um "pescoço" flexível que permita a mobilidade. Para reduzir o brilho, certifique-se que a lâmpada esteja abaixo do nível do olho; para iluminação de tarefa/leitura é recomendado uma lâmpada de 60 watts colocado a $30 \mathrm{~cm}$ longe da tarefa (BAKKER, 2013).

\section{Iluminação direta}

Deve-se ressaltar os detalhes que sejam de interesse, tais como escadas, cartazes, números, painéis, indicativos, plantas, utilizando luzes diretas sobre eles, sem produzir reflexos (FRESTEIRO, 2005). Os níveis devem ser de 500 lux (ESCOBAR, 2011). 


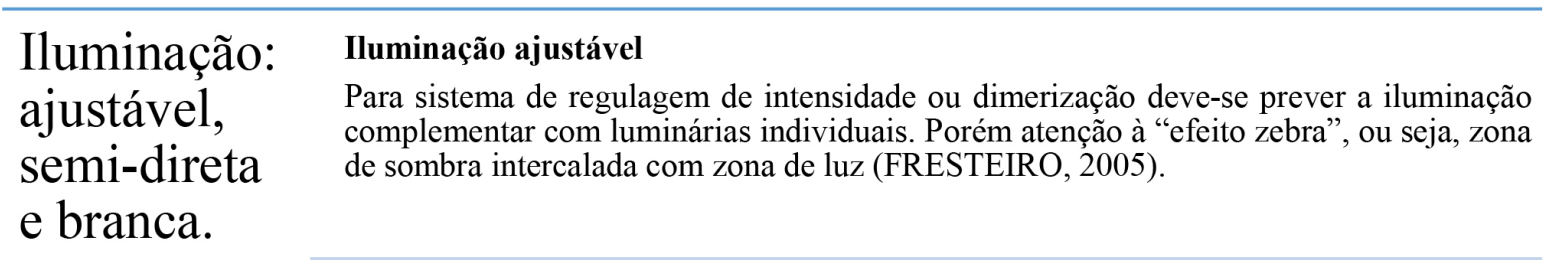

\title{
Iluminação semidireta
}

A luz semi-direta, que não incide em sua totalidade em uma área determinada e proporciona menos sombras e ofuscamentos é preferível. (FRESTEIRO, 2005).

\author{
Iluminação branca \\ Lâmpadas fluorescentes e LED de espectro total são mais confortáveis porque realça \\ cores e contraste, principalmente para os indivíduos com degeneração macular \\ (BAKKER, 2013). Luz branca fria (com um tom azulado) é excelente para tarefas \\ visuais e proporciona iluminação geral difusa enfatizando as cores que contem azul e \\ que proporcionem iluminação uniforme (FRESTEIRO, 2005).
}

Fonte: os autores (2016)

\section{Considerações finais}

Ao abordar os fatores humanos e sistemas de tecnologias assistivas no contexto do design de interiores, percebe-se constantes melhorias que devem ser implementadas para favorecer qualidade de vida a pessoas que possuem deficiência, neste caso, visual. Sendo assim, o design centrado no usuário permite identificar as necessidades e limitações e promover segurança e eficácia na realização das atividades.

Neste trabalho foi possível organizar e construir um conjunto de diretrizes para projetos de iluminação de modo a incluir pessoas com baixa visão. O intuito foi pesquisar e utilizar as diferentes normas, modelos e recomendações de diversos autores e sintetizá-las em um único trabalho, para que seja possível a aplicação destas diretrizes. Permite assim que profissionais possam se basear nestas diretrizes e projetar ambientes para pessoas portadoras de visão subnormal, e que estas possam exercer suas atividades, e frequentar ambientes internos e sociais percebendo o espaço de maneira mais plena possivel.

É necessário enfatizar a importância do Design e da Arquitetura na criação de espaços inclusivos que ofereçam conforto e autonomia, evitando possíveis riscos e acidentes devido à falta de planejamento no ambiente. Para pesquisas futuras pretende-se fazer uma maior captura dessas informações através de um estudo de caso, observando se estas diretrizes são contempladas em espaços internos públicos e, se não, devem ser ampliadas, de modo a proporcionar uma maior inclusão social.

\section{Agradecimento}

Os autores deste artigo agradecem a PROMOP, Programa de Bolsas de Monitoria de Pós-Graduação, que possibilita a bolsa de estudos durante o período do mestrado e ao Programa de Pós-Graduação em Design pelo apoio na realização da pesquisa. 


\section{Referências Bibliográficas}

ASSOCIAÇÃO BRASILEIRA DE NORMAS TÉCNICAS (ABNT). NBR ISO/CIE 8995:1 - Iluminação de ambientes de trabalho Parte 1: Interior. Rio de Janeiro: ABNT, 2013.

ALLAN, Jim; KIRKPATRICK, Andrew; HENRY, Shawn Lawton. Accessibility requeriments for people with low vision. W3C First Public Working Draft 17 march 2016. Disponívelem: https://www.w3.org/TR/low-vision-needs/\#informative-references. Acesso em: 19 out 2016.

ANDRADE, Pablo Martín. Accesibilidad para personas com cegueira y deficiência visual. Madrid, 2003.

BAJA VISIÓN ÁNGEL BARAÑANO, Centro especializado enla tratamento de la Baja Visión. Madrid, Disponível em: http://www.baja-vision.org/ acesso em: 24 out 2016.

BAKKER, R. Household tips for people with low vision. Cornell University Program for Environmental Geriatrics, 2013.

BONATTI, JA, SAMPAIO MW, BONATTI FAZ, SANTOS MCL, KARA-JOSÉ N. Responsabilidade social em oftalmologia: interdisciplinaridade e inclusão na visão subnormal. RevMed (São Paulo). 2007 out.-dez. 86(4):195-200.

Decreto no 5.296/04, de 02 de dezembro de 2004.Regulamenta as Leis nos 10.048 , de 8 de novembro de 2000 , que dá prioridade de atendimento às pessoas que especifica, e 10.098, de 19 de dezembro de 2000, que estabelece normas gerais e critérios básicos para a promoção da acessibilidade das pessoas portadoras de deficiência ou com mobilidade reduzida, e dá outras providências. Disponível em: <https:// www.planalto.gov.br/ccivil_03/_Ato2004-2006/2004/Decreto/D5296.htm>. Acesso em: 13 out. 2016.

Lei 38/2004, de 18 de agosto de 2004. Define as bases gerais do regime 330077 jurídico da prevenção, habilitação, reabilitação e participação da pessoa com deficiência. Disponível em:<http://www.parlamento.pt/ActividadeParlamentar/Paginas/DetalheDiplomaAprovado.aspx?BID=5474>. Acessoem: 13 out. 2016.

Brunnström, Gunilla, et al. Quality of light and quality of life-the effect of lighting adaptation among people with low vision. OphthalmicandPhysiologicalOptics, 2004: 274-280.

Cartilha do Censo 2010 - Pessoas com Deficiência / Luiza Maria Borges Oliveira / Secretaria de Direitos Humanos da Presidência da República (SDH/PR) / Secretaria Nacional de Promoção dos Direitos da Pessoa com Deficiência (SNPD) / Coordenação-Geral do Sistema de Informações sobre a Pessoa com Deficiência; Brasília : SDH-PR/ SNPD, 2012.

CONFORTO, E.C.; AMARAL, D.C.; DA SILVA, S.L. Roteiro para revisão bibliográfica sistemática: aplicação no desenvolvimento de produtos e gerenciamento de projetos. In: 80 CONGRESSO BRASILEIRO DE GESTÃO DE DESENVOLVIMENTO DE PRODUTO, 2011, Porto Alegre.

COSTA, Andreia Patrícia Vidinha Frias da. Proposta de Guia de Boas práticas na integração de pessoas cegas e com baixa visão nos locais de trabalho. 2012. 216 p. Dissertação de mestrado (Engenharia de segurança e higiene ocupacionais) - Faculda- 
de de Engenharia. Universidade do Porto, Porto.

EBAY. Adjustable Flexible Hug Book 4 LED Light Neck Night Lamp Torch For Study Reading. Disponível em: http://www.ebay.ca/itm/Adjustable-Flexible-Hug-Book-4-LED-Light-Neck-Night-Lamp-Torch-For-Study-Reading\%20 /232128208434?hash=item360beb5e32:g:A48AAOSwt5hYbwDY. Acesso em: 20 abr 2017.

ESCOBAR, Geanine Vargas. O Conservador em conflito: A lluminação em Museus com Proposta para Inclusão de Pessoas com Deficiência Visual. 2011. 58 p. Monografia (Graduação) - Curso de Bacharelado em Conservação e Restauro da Universidade Federal de Pelotas, Pelotas, RS.

EUROPEAN STANDARD (EN 12464-2). Lighting of work places - part 2: Outdoor work places. Bruxelas: CEN, 2007.

FAIA, Stuart L. Knoop. Architecture for low vision. National institute of building sciences low vision design committee symposium, 10 jan 2016. Disponível em: https:// www.brikbase.org/content/architecture-low-vision-site-building-and-interior-design. Acesso em: 19 out 2016.

FERENHOF, HelioAisenberg; FERNANDES, Roberto Fabiano. Desmistificando a revisao de literatura como base para redacao cientifica: métodos sf. Revista ACB, v. 21, n. 3, 2016.

FRESTEIRO, R. H. Laboratory of experimental lighting for low vision people. In: InternationalCongress Series. Elsevier, v. 1282, 2005. p. 1011-1015.

. La iluminácion de losespacios como parâmetro de acessibilidade para personas com baja visión. Tese de doutorado, Universidad Politécnica de Madrid, 2002.

. Iluminação inclusiva, ou eliminando barreiras lumínicas. In: Bregatto, P.R. Documentos de arquitetura - Traços $\&$ Pontos de vistas, Porto Alegre: Editora da Ulbra, 2005, p. 190-201.

A influência da iluminação nos ambientes acessíveis: identificando barreiras. In: ORNSTEIN, S. W; ALMEIDA PRADO, Adriana R. de; LOPES, M. E. Desenho universal: caminhos da acessibilidade no Brasil, São Paulo: Annablume, 2010, p. 267-278.

GIL, Mara. Deficiência visual. Secretaria da educação à distância. Brasília: Brasil, 2000. Disponível em: http://www.dominiopublico.gov.br/download/texto/me000344. pdf. Acesso em: 22 ago 2016.

LIGHT RESEARCH CENTER. Advanced the effective use of light for society and the environment. Disponível em: http://www.lrc.rpi.edu/programs/NLPIP/lightingAnswers/halogenTorchiers/quest_seven.asp. Acesso em: 20 abr 2017.

LAUAR, Ana Clara Fernandes. Comparação entre a percepção e a normatização sobre iluminação em ambientes ocupacionais: estudo de caso em uma empresa florestal. 2012. 96p. Dissertação de mestrado em Desenho Industrial (área de concentração: Desenho do Produto; linha de pesquisa: Ergonomia)- Faculdade Artes, arquitetura e comunicação da Universidade Estadual Paulista "Julio de Mesquita Filho", Bauru.

MIZIARA, Paulo. Auxílios não ópticos para pessoas com baixa visão. 2015. Disponível em: http://www.acessibilidadenapratica.com.br/textos/auxilios-nao-opticos-para-baixa-visao/,acesso em: 19 out. 2016. 
RIAZI, Abbas et al. Home modification guidelines as recommended by visually impaired people.Journalofassistive Technologies. v. 6, n. 4, 2012, p. 270-284

Saberes e práticas da inclusão: Desenvolvendo competências para o atendimento às necessidades educacionais especiais de alunos cegos e de alunos com baixa visão. [2. ed.] / coordenação geral SEESP/MEC. - Brasília: MEC, Secretaria de Educação Especial, 2006. 208 p. (Série: Saberes e práticas da inclusão)

SONZA, Andréa Poletto. (org) et al. Necessidades educacionais especiais. In: SONZA, Andréa Poletto; FÉO, Fabíola; PAGANI, Josiane. Acessibilidade e tecnologia assistivia: pensando a inclusão sociodigital de pessoas com necessidades especiais. $2^{2}$ ed. Bento Gonçalves, 2013 n. 67-165.

TURAL, Elif; TURAL, Mehmedalp. Luminance contrast analyses for low vision in a sênior living faciluty: A proposal for na HDR image-based analysis tool. Building and Environment, v. 81, 2014, p-20-28.

WRIGHT, M; HILL, S; COOK, G. Office task lighting: A user study of six task lights by five workers with low vision. The British journalof visual impairment, 1999, p. 117-120. 